sciendo

DOI:10.2478/sjpna-2022-0005

\title{
DIRECTIONS OF DEVELOPMENT \\ OF THE AUTONOMOUS UNMANNED UNDERWATER VEHICLES. A REVIEW
}

\section{Mateusz Orłowski}

Polish Naval Academy, Faculty of Mechanical and Electrical Engineering, Śmidowicza 69 Str., 81-127 Gdynia, Poland; e-mail: m.orlowski@amw.gdynia.pl

\begin{abstract}
In the paper review of unmanned underwater vehicle (AUV) is presented. The description of main systems is depicted with focus on autonomous single vehicle as well as a swarm. As a consequence of development of AUV technology, research centers are focused on issues related to increasing the degree of their autonomy. Nowadays, mostly navigation and communication as well as high-efficient propeller systems are being developed. There are problems linking this issues. Their solutions includes development of new control laws containing algorithms to prevent collisions - for unmanned vehicles with elements of the underwater environment and for several underwater vehicles cooperating with each other in a swarm.
\end{abstract}

Key words:

Unmanned Underwater Vehicles, Remotely Operated Vehicles, Autonomous Underwater Vehicles, Biomimetic Underwater Vehicles

Research article

(C) 2021 Mateusz Orłowski This is an open access article licensed under the Creative Commons Attribution-NonCommercial-NoDerivatives 4.0 license (http://creativecommons.org/licenses/by-nc-nd/4.0/) 


\section{INTRODUCTION}

Presented in Fig. 1 torpedo shaped construction was presented by Robert Whitehead in 1866, is considered to be the first prototype of the Unmanned Underwater Vehicles (UUV).

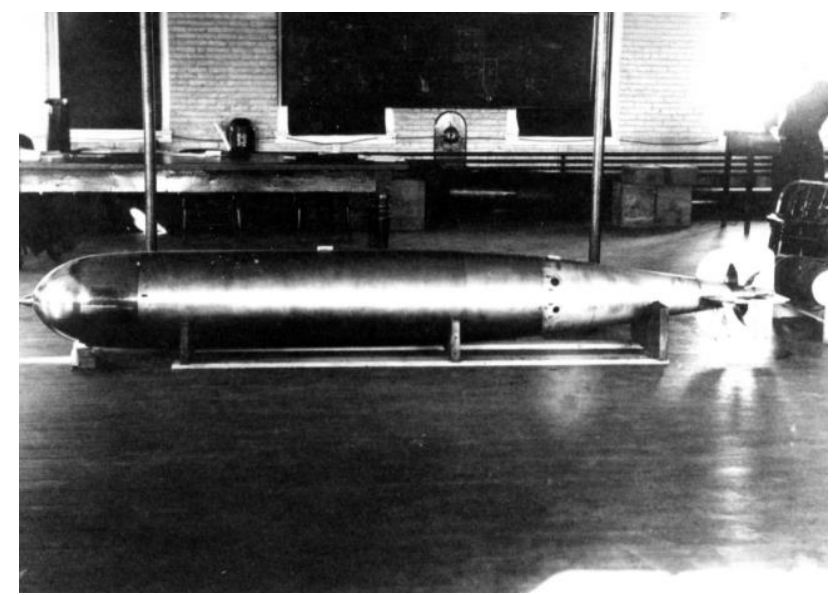

Fig. 1 A MK1 Whitehead torpedo [23]

This discovery was a milestone in the process of development of the underwater vehicle technology. However, the real development of the UUV began in 1957 due to the beginning of the project of an autonomous underwater research vehicle - Self Propelled Underwater Research Vehicle (SPURV). The first fully functional version (SPURV I) was created in the mid-70s. Weighing $480 \mathrm{~kg}$, controlled from the surface with acoustic waves, the vehicle was able to move at a speed of $2.2 \mathrm{~m} / \mathrm{s}$ at a depth of $5.5 \mathrm{~km}$. Based on the pressure measurement at a specific depth, it was possible to plan the vehicle movement along a specific isobar or between two selected reference levels. The control system and drive used allowed for maneuvering in a vertical plane at angles up to 50 degrees [1].

Further research on the UUV technology consisted primarily in increasing the range, time and depth of their operations.

Nowadays, scientists are focused on the problems connected with the fully autonomous vehicles which solutions will allow to eliminate the need for continuous human control of their parameters. The development of the UUVs consists mainly in increasing the range, time and depth of their operations. Scientists have begun research on fully autonomous vehicles that would completely eliminate the need for continuous traffic parameters control by the human operator. This led to natural division of UUVs into Remotely Operated Vehicles (ROVs) and Autonomous Underwater Vehicles (AUVs) [1].

The requirements for modern AUVs lead to the need of development of technologies that increase their autonomy in performing tasks with a wide spec- 
trum of diversity in an increasingly demanding environment. Therefore, research is currently being carried out, among others above:

- highly efficient power sources that provide energy for the power unit, sensors and control unit,

- control and navigation systems to detect and avoid obstacles and hazards,

- an effective and reliable method of communication between vehicles,

- measurement systems connected with the purpose of a given AUV [2],

- high-efficient propulsion systems [12].

Excellent example being the final result of work on the above-mentioned issues is the Echo Voyager (Fig. 2), developed and constructed by the Boeing company. It is classified as long-range Extra Large Unmanned Undersea Vehicle (XLUUV).

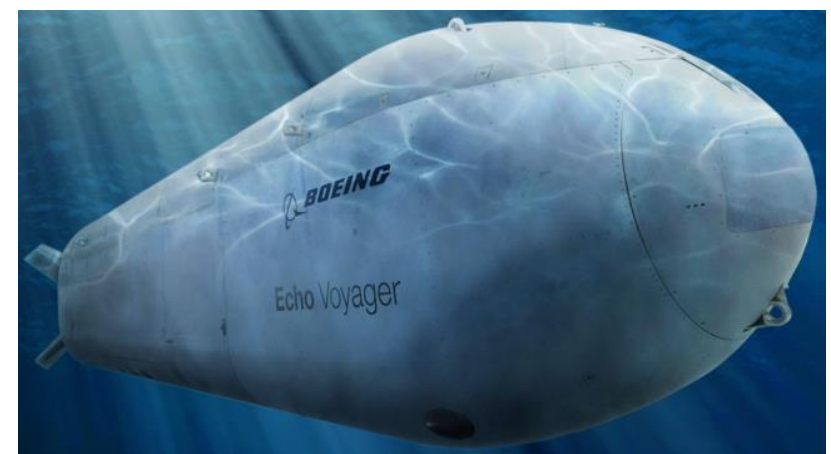

Fig. 2 Boeing Echo Voyager - Extra Large Unmanned Undersea Vehicle [24]

On-board equipment, weighing $45630 \mathrm{~kg}$ AUV, includes over 20 tons of various types of systems ensuring full autonomy for several months and covering total distance of $6500 \mathrm{~nm}$. This was done by optimizing the use of highly efficient $18 \mathrm{~kW}$ power source, which also supplies energy to the on-board measurement and propulsion systems. Basic Echo Voyager's parameters are presented in Tab. 1.

Tab. 1 Summary of basic Echo Voyager's parameters [2, 24]

\begin{tabular}{|l|l|}
\hline Parameters & Value \\
\hline Weight & $45630 \mathrm{~kg}$ \\
\hline Envelope (no payload) & $15,5 \times 2,5 \times 2,6 \mathrm{~m}$ \\
\hline $\begin{array}{l}\text { Envelope (with a payload sec- } \\
\text { tion) }\end{array}$ & $25,9 \mathrm{~m}$ \\
\hline Maximum depth & $3300 \mathrm{~m}$ \\
\hline Maximum speed & $4,1 \mathrm{~m} / \mathrm{s}$ \\
\hline Altitude stability & $0,25 \mathrm{~m}$ \\
\hline Depth stability & $0,3 \mathrm{~m}$ \\
\hline
\end{tabular}




\begin{tabular}{|l|l|}
\hline Unaided position accuracy & $\begin{array}{l}0,15 \% \text { distance trav- } \\
\text { elled }\end{array}$ \\
\hline Aided position accuracy & $2,3 \mathrm{~m}$ \\
\hline Operation time & 6 months \\
\hline
\end{tabular}

However, the possibility of extending the scope of on-board equipment with various measurement systems and sensors would make AUVs more universal. On the one hand, the progressing miniaturization in the near future will certainly enable the design and implementation of a such vehicle. But, on the other hand, one of the most important problems in the vast majority of AUVs projects is the limited budget for equipment and research. The development of modern technologies brings with it an increase of costs, as well as the degree of system complexity, which not all users will be able or willing to afford.

For this reason, currently research on vehicles specialized in specific solutions is dominating. AUVs used both in the civilian and military environments are designed to perform strictly defined tasks. They can include, among others:

- inspection of the technical condition of cooling tanks for reactors in nuclear power plants, as well as tanks intended for the storage of spent nuclear fuel [2],

- $\quad$ searching for damage to the hulls and elements of their propulsion system under the water surface, as well as leaks from underwater tanks and fossil fuel pipelines, damage to optical fibers and power cables [2],

- performing an accurate scan with high resolution of the bottom topography [3],

- $\quad$ study of marine fauna and flora at very great depths, including the regions of both Earth's poles, and the characteristics of the movements of water masses in the areas under consideration [5],

- to gain an advantage at the tactical, operational and strategic level in the theater of military operations [6], etc.

Taking into account the multitude of factors determining the diversification of the degree of difficulty and the nature of the mission, attention should be paid to the constantly emerging new problems limiting the possibilities of using AUV in a specific situation. The latest research mainly covers development in the areas of navigation, communication, simulation, management and control systems [4].

\section{Navigation systems}

Autonomous performance of tasks by the AUV is limited by correct operation of systems implemented on its board.

For this reason, numerous research centers take up a challenge of increasing their reliability and accuracy in the most possible optimal way.

According to the requirements given above the electromagnetic wave systems seems to be the best solution. However, propagation of electromagnetic waves in underwater environment is very weak. Due to its properties and use of the Global 
Navigation Satellite Systems, such as Global Positioning System (GPS), Global Navigation Satellite System (GLONASS) and Galileo in the AUV navigation systems is extremally limited or even impossible. For this reason, other methods of navigation based on Inertial Navigation Systems (INS) or Doppler Navigation Systems (DNS) are used $[2,3,19]$. Such solutions were used, for example, in the Autosub6000 project [7], where the positioning error was up to $5 \mathrm{~m} / \mathrm{h}$ of operation.

The other way of navigation is modeling dynamics of motion with 6 DoF (Degrees of Freedom) and mathematical determination of object's trajectory. The advantage of this approach is resistance to the influence of external disturbances, because navigation is based on the simulation results and do not rely on the measurement of environmental parameters with the independent sensors. The disadvantages include the inability to use favorable external factors allowing optimization of energy use. Lack of resistance of the control model to incorrect values of implemented parameters (assumed in advance for the simulation purposes) may lead to incorrect calculations of the trajectory of the movement and, as a consequence, to a dangerous situation or preventing of the proper task realization.

However, classic DNS, INS or simulating methods are still insufficient if the AUV's mission requires more precise navigation data. A solution of the problem could be a method known as map-based localization, but it is also limited due to the necessity of possession of the explored area maps [4].

As a result of the listed above limitations it is very difficult to design a AUV with a high degree of autonomy. Additionally, it is necessary to take into account limited ability to simultaneously energize the on-board sensors that supply data for navigation, control and communication systems.

Solving navigation problem seems to be almost impossible or at least very difficult if very precise and consequently expensive INS or DNS that rely on multiple sensors are not used. However, the development of the MEMS (microelectromechanical systems) technology and mentioned below two main methods that increase accuracy, decrease cost and reduce size of basic navigation systems:

- acoustic localization,

- visual localization [2].

\subsection{Acoustic localization}

Acoustic localization includes Acoustic Beacons, Simultaneous Localization and Mapping (SLAM) and Doppler Velocity Logs (DVLs).

The Acoustic Beacon method consists in determining position by measuring time delay between two transponders (one of them is the AUV and the second is the space-fixed device) azimuth of the transponder [7]. Accuracy of such a system depends on whether the device has been positioned at the exact point with known coordinates - discrepancies will lead to incorrect positioning of the AUV.

Standard Doppler Velocity Log (DVL) sends four acoustic beams shifted relative to each other by some known downward angle. The Doppler shift is proportional to the speed the vehicle above the seabed. DVL vehicle speed and heading provided by the IMU or the AHRS system allows to indicate certain AUV's position. 
The SLAM method consists in developing a map of the area surrounding AUV. Main benefit of this method is that AUV's initial location is not necessary. Characteristic points marked on the map serve as reference points in the further navigation process. The criterion for selecting an appropriate sensor depends on speed or range of the scan. Then mainly LiDAR (Light Detecting And Ranging), sonar or magnetic SLAM sensors are used [8]. Nowadays, SLAM based navigation systems dominate in fully autonomous vehicle navigation systems.

\subsection{Visual localization}

The visual method uses cameras as sensors. Main challenge of this method is to develop an algorithm for the purpose of interpreting images from the camera in order to use it for navigation - determining route by recognizing obstacles along the way.

Recent technological advances have accelerated both the miniaturization and the quality of cameras. Vision-based localization systems are not sensitive to the interference phenomena that are common in acoustic and electromagnetic approaches.

Underwater conditions related to the physics of light phenomena (for example light absorption), as well as physical or chemical haze can significantly impede visual detection.

Despite the limitations, visual localization has promising effects for tasks where good visibility conditions are expected [2].

\section{Communication systems}

There are similar problems for underwater communication of cooperating AUVs - environment properties dumps electromagnetic wave propagation and the range of possible communication between cooperating vessels is limited.

The acoustic wave is characterized by much better propagation properties than the electro-magnetic wave and this is the reason why the acoustic-based systems are most reliable means of positioning [2]. They are commonly used due to higher range than optical solutions.

The greatest efficiency would come from combining short-range optical systems with long-range acoustic systems. However, usually the use of acoustic devices is very limited as they have quite large dimensions and weight, while the trend is to minimize the dimensions of the AUV itself [18].

Other important issue is to increase autonomy of AUVs, which could avoid problems in a case of limited possibilities in the data exchange. This is achieved by the development of algorithms that allow to calculate the optimal trajectory with continuously and randomly changing parameters of the surrounding environment, including algorithms related to the avoidance of obstacles and collisions (for example in the case of swarm AUV systems). Optimalization problem is also connected with the energy storage efficiency - it is necessary to provide highly efficient power sources supplying the propulsion and the on-board navigation, control and com- 
munication systems. Additionally, the control algorithms should include a function that will minimize the energy consumption while driving in designed routes.

Underwater communication systems rely on acoustic or optical technology. There is not any ideal solution. Both of them have their own significant advantages and disadvantages. Low cost and significantly higher (comparing to the acoustic method) data transmission speed contribute to advantages of using the optical method. Its main disadvantage is short range, which depends, for example on the contamination of the medium, random obstacles in the communication path and the possibility of a wave breakage and reflection [9]. For this reason, the use of optical method in data transfer is mainly used in the area of application of AUV swarms cooperating with each other at short distances.

In other areas, only acoustic methods have been used, and they are the majority of developed methods of underwater communication. Range of frequencies used in this method are between $10 \mathrm{~Hz}$ and $1 \mathrm{MHz}$, with the lower limit resulting from inability to use acoustic waves lower than $10 \mathrm{~Hz}$ at greater depths, while waves with frequencies above $1 \mathrm{MHz}$ are absorbed very quickly [10].

The properties of propagation under water are influenced mainly by:

- noise, resulting from turbulent water movements, ship movements, waves and temperature changes (thermal noise),

- multi-channel media, the problem of which are fading and three types of interference: inter channel interference, inter symbol interference and inter carrier interference,

- Doppler effect [10].

Dynamic development of information transmission technology through electromagnetic induction has been observed for several years. Its advantage over other methods consists mainly in a much smaller impact on the above-mentioned disturbances and the possibility of obtaining very high data transfer speeds.

However, its main limitation is its very high sensitivity to changes in electrical conductivity of medium, which has a direct impact on the range of data exchange. The example discussed in [11] shows difference in case of aquatic environment. A small, low-power device can reach a maximum range of 20 meters for drinking water, but only 1 meter for sea water.

\section{Collision avoidance systems}

Additional difficulty linked with navigation and communication issues is collision avoidance problem. It combines necessity to use appropriately sensitive sensors and development of software that allows to recognize the "hostile" object with sufficient accuracy and develop an appropriate solution ensuring safety for the AUV.

The first approach to the mentioned above problem is deliberative or senseplan-act approach - consists in determining the entire route from the current AUV position to a given point [16]. Its main disadvantage is that it can be used only in case of offline global path planning or when fast decision-making related to possibility of negative collision risk is not required. 
The second approach is called reactive or sense-act approach [16]. Contrary to the previous one, it is based on current measurements carried out by on-board AUV sensors. Its advantage is speed of action and high priority of response to random threat. However, as a consequence, this property may adversely affect the optimization of AUV route mapping in a wider perspective. For this reason, it has been used to perform tasks in dynamically changing environment where the risk of collisions is high.

Example of collision avoidance system solution used in AUV is the use of hydrophones to measure acoustic wave parameters as useful information for Biomimetic Underwater Vehicles (BUV) in the international SABUVIS project. A passive system based on two HTI-96-MIN hydrophones with omnidirectional characteristics was used. Sensors are situated in the upper part of the BUV with 1.25 meter distance between each other [17]. The results of the research on the developed solution are presented in publication [15].

\section{Propulsion systems}

The most common type of AUV drives uses rotors as propellers. However, efforts to increase efficiency made it necessary to depart from standard solutions. One of them, studied and developed at the Polish Naval Academy, is undulating propulsion system used in Biomimenic Underwater Vehicles.

One of the main reasons for the efforts to develop this type of propulsion is their high efficiency resulting directly from the evolution of fish, which has perfected them for millions of years. They also produce less acoustic noise than classical propellers $[12,13]$.

Maximizing efficiency requires use of very complex algorithms for controlling drive system. Due to characteristics of the environment, it is necessary to conduct laboratory tests in water tunnels and to use specialized computer software in numerical simulation of propeller's dynamics [14].

Imitating the movement of the fish fin can be done mainly by using two types of drives [13]:

- a single fin made of flexible material - Fig. 2,

- a set of interconnected rigid elements whose change of mutual position imitates the shape assumed in natural conditions by tail fin - Fig. 3 .

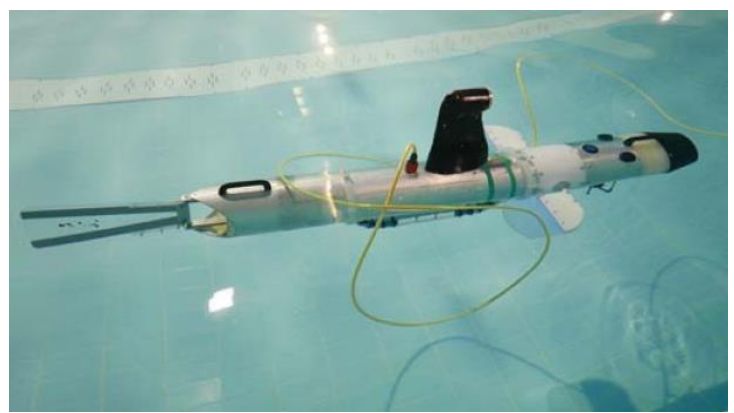

Fig. 2 The BUV with one tail flexible fin [13] 


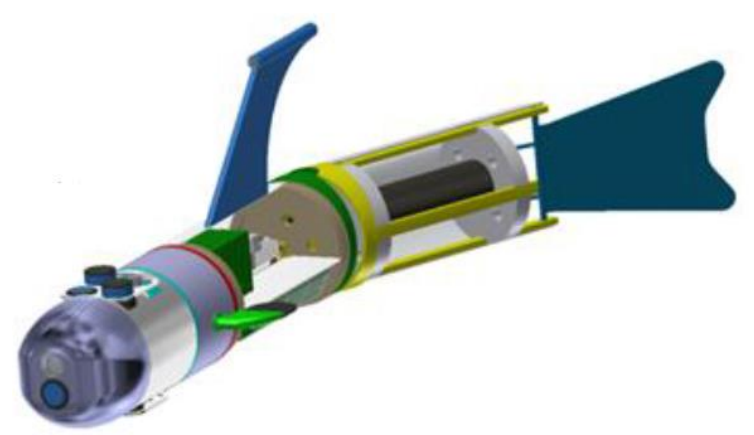

Fig. 3 The BUV with tail fin made of inter-connected parts [20]

A hybrid combination of listed above types of drives can also be used. This solution was used, among others in the CyberFish project shown in Fig 4.

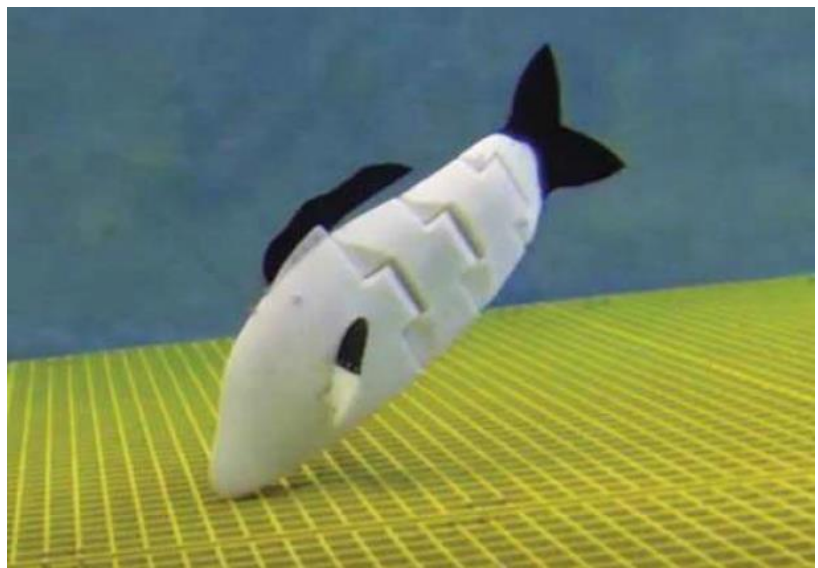

Fig. 4 The CyberFish with tail fin made of three interconnected rigid elements and end-part made of flexible material [12]

\section{Model verification and testing}

Increasing autonomy of the AUVs is multi-dimensional process and entails development of the technologies mentioned in the previous sections of this article, as well as development of new control algorithms and mission management systems.

The marine environment is very demanding due to its properties - there are many variable factors of stochastic nature, impact of which on the control process significantly hinders development of optimal control algorithm for AUVs.

Modeling dynamics of AUV motion also requires taking into account the changes affecting forces and moments acting on a moving object in the water environment, parameters of which may be nonlinearly variable in time. Developed solutions should be tested in right order, initially based on computer simulation, and after positive verification of theoretical model, tested in real conditions. 
First step is called "software in the loop" method and it uses computer simulators. One of the most popular examples is the Gazebo simulator, which allows to verify the developed models of robots and their control algorithms for many scenarios. Gazebo is based on the open source Unix system. Its great advantage is sharing of developed solutions by users from around the world. It allows for more dynamic development of research in the field of underwater robotics.

The second stage of research is the "hardware in the loop" method - the implementation of programs describing development of algorithms on a real model and examining its behavior in real conditions. It is also a step that allows you to determine real energy expenditure used by propulsion, control systems and other AUV on-board systems. Achieving maximum time and scope of operations requires development of energy storage technology and its optimal use, taking into account useful properties of the marine environment (including, for example, energy of sea currents).

\section{Conclusion}

Requirements for today's AUVs are constantly increasing. One of the most important is reliability, which means that a specific task can be successfully completed with the highest probability. This is achieved both through development of manufacturing technology of individual components of the AUVs' on-board systems - hardware, and improvement of software and control algorithms - software.

Fig. 4 shows steady increase in the number of published AUV-related papers presented in 2001-2020. There is a visible upward trend, which proves the growing interest in AUV and increasing intensity of development works carried out by research centers over the world.

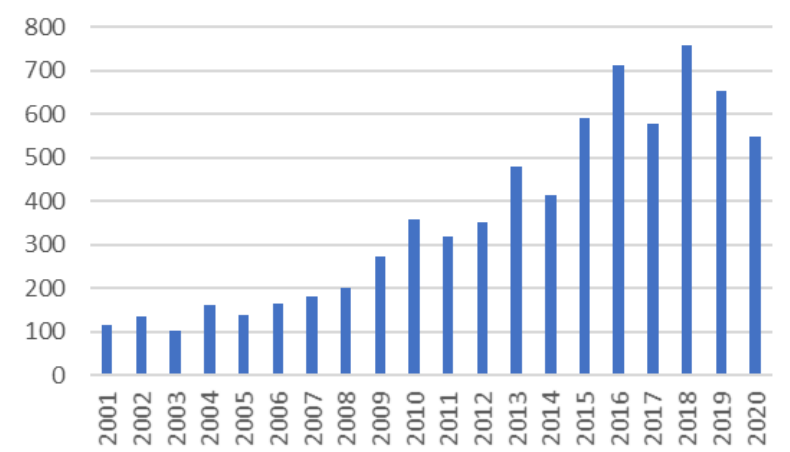

Fig. 4 Number of AUV papers published in 2001-2020 [21]

From the beginning of the 1980s to 2000, just over 800 AUV-related papers were published. In turn, since the beginning of the 21st century, their total number has increased by over 7200, which means that development of AUV is a fairly young field of science with a dynamic pace of development in the near future. 
Additionally, dynamic development of AUV swarm technology has been observed in recent years, the use of which brings many benefits. The most important include shortening time of performing tasks and ensuring greater reliability of the system - unlike single use robots, the loss of one vehicle from a swarm does not cause immediate failure.

One of the most famous research projects dealing with swarm issues is SWARMs platform (Smart and Networking Underwater Robots in Cooperation Meshes). Its goal is to develop the open-architecture platform that allows for cooperation of individual elements of the swarm, ensuring easy reconfiguration for needs of tasks currently performed. The areas of work on the platform are divided into nine areas:

- Management;

- Autonomous operations design - supervising and controlling the platform design process;

- Coordination Architecture and Specification - implementation of generally available solutions for the platform - both hardware and software;

- Environment Recognition and Sensing - development of AUV measurement systems;

- Communication and Networking - development of high-speed acoustic communication;

- Vehicle embedded architecture and task planning - development of management systems with the possibility of reconfiguring mission parameters in real time;

- Autonomous navigation and semi-autonomous manipulation - increasing the functionality of the navigation system by using data from various system components;

- Demonstrator: Definition, Integration, Verification and Validation - verification of the developed system solutions;

- Dissemination, Exploitation and Standardization [22].

Total cost of the project in 2015-2018 was $€ 17300000$. Thirty partners from ten European countries were involved in its development. This shows that the demand for technology increasing independence of the AUV swarming system is very high.

The examples of development directions cited above show that striving to increase autonomy is associated with a multidimensional process consisting in the simultaneous improvement of existing technologies and an attempt to develop completely new and innovative solutions.

\section{References}

[1] Christopher von Alt, Autonomous Underwater Vehicles, Woods Hole Oceanographic Institution, 2003

[2] Simon Watson, Daniel A. Duecker, Keir Groves, Localisation of Unmanned Underwater Vehicles (UUVs) in Complex and Confined Environments: A Review, Sensors, 2020

[3] Jinyeong Heo, Junghoon Kim, Yongjin Kwon, Technology Development of Unmanned Underwater Vehicles (UUVs), Journal of Computer and Communications, 2017 
[4] Yvan R. Petillot, Gianluca Antonelli, Giuseppe Casalino, Fausto Ferreira: Underwater Robots, From Remotely Operated Vehicles to Intervention-Autonomous Underwater Vehi-cles, IEEE Robot. Autom. Magazine, 2019

[5] David K.A. Barnes, Andrew Clarke, Antarctic marine biology, Current Biology, 2011

[6] Ronald O'Rourke, Navy Large Unmanned Surface and Undersea Vehicles: Background and Issues for Congress, Congressional Research Service, 2020

[7] Ziye Zhou, Yanqing Jiang, Ye Li, Cao Jian, Yeyi Sun, A single acoustic beacon-based positioning method for underwater mobile recovery of an AUV, International Journal of Advanced Robotic Systems, September-October 2018, pp. 1-10

[8] Guangchao Hou, Qi Shao, Bo Zou, Liwen Dai, Zhe Zhang, Zhehan Mu, Yadong Zhang, Jingsheng Zhai, A Novel Underwater Simultaneous Localization and Mapping Online Algorithm Based on Neural Network, International Journal od Geo-Information, 2020, pp. 2-5

[9] B. Pranitha, L. Anjaneyulu, Analysis of Underwater Acoustic Communication System Using Equalization Technique for ISI Reduction, Procedia Computer Science, No. 167, 2020, pp. 1128-1138

[10] B. Pranitha, L. Anjaneyulu, Research Trends in Underwater Communications - A Technical Survey, International Conference on Communication and Signal Processing, 2016

[11] Ian F. Akyildiz, Pu Wang, Zhi Sun, Realizing Underwater Communication through Magnetic Induction, IEEE Communication Magazine, 2015

[12] Paweł Piskur, Piotr Szymak, Zygmunt Kitowski, Leszek Flis, Influence of Fin’s Material Capabilities on the Propulsion System of Biomimetic Underwater Vehicle, Polish Maritime Research, Vol. 27, No. 4, 2020, pp. 179-185

[13] Karolina Jurczyk, Paweł Piskur, Piotr Szymak, Parameters Identification of the Flexible Fin Kinematics Model Using Vision and Genetic Algorithms, Polish Maritime Research, Vol. 27, No. 2, 2020, pp. 39-47

[14] Paweł Piskur, Piotr Szymak, Leszek Flis, Joanna Sznajder, Analysis of a Fin Drag Force in a Biomimetic Underwater Vehicle, Naše More, Vol. 67, No. 3, 2020, pp. 192-198

[15] Paweł Piskur, Piotr Szymak, Krzysztof Jaskólski, Leszek Flis, Marek Gąsiorowski, Hydroacoustic System in a Biomimetic Under-water Vehicle to Avoid Collision with Vessels with Low-Speed Propellers in a Controlled Environment, Sensors, 2020, 20, 968

[16] Tomasz Praczyk, Neural collision avoidance system for biomimetic autonomous underwater vehicle, Soft Computing, No. 24, pp. 1315-1333

[17] Paweł Piskur, Marek Gąsiorowski, Digital Signal Processing for Hydroacoustic System in Biomimetic Underwater Vehicle, Naše More, Vol. 67, No. 1, 2020, pp. 14-18

[18] Tomasz Praczyk, Detection of Land in Marine Images, International Journal of Computational Intelligence Systems, Vol. 12, No. 1, 2019, pp. 273-281

[19] Tomasz Praczyk, Using Neuro-Evolutionary Techniques to Tune Odometric Navigational System of Small Biomimetic Autonomous Underwater Vehicle - Preliminary Report, Journal of Intelligent \& Robotic Systems, 2020, pp. 363-376

[20] Tomasz Praczyk, Piotr Szymak, Krzysztof Naus, Leszek Pietrukaniec, Stanisław Hożyń, report on research with biomimetic autonomous underwater vehicle - navigation and autono-mous operation, Scientific Journal of Polish Naval Academy, Vol. 2, 2018

[21] https://ieeexplore.ieee.org/

[22] http://www.swarms.eu/approach.html

[23] https://navalunderseamuseum.org/whitehead/

[24] https://www.boeing.com/resources/boeingdotcom/defense/autonomous-systems/echovoyager/echo_voyager_product_sheet.pdf 